\title{
Recent 5-Year Trend of Endoscopic Retrograde Cholangiography in Korea Using National Health Insurance Review and Assessment Service Open Data
}

\author{
Jin Myung Park ${ }^{1}$, Chang Don Kang ${ }^{1}$, Jong-chan Lee ${ }^{2}$, Jin-Hyeok Hwang ${ }^{2}$, and Jaihwan Kim² \\ ${ }^{1}$ Department of Internal Medicine, Kangwon National University Hospital, Kangwon National University School of Medicine, Chuncheon, and \\ ${ }^{2}$ Department of Internal Medicine, Seoul National University Bundang Hospital, Seoul National University College of Medicine, Seongnam, \\ Korea
}

Background/Aims: The utilization of endoscopic retrograde cholangiopancreatography (ERCP) is variable and largely dependent on a patient's age, sex, and region. Herein we analyzed the publicly available Health Insurance Review and Assessment (HIRA) database with the aim of understanding the current status and trend of ERCP use in Korea. Methods: Between 2013 and 2017, information regarding ERCP was acquired from the HIRA database using the procedural codes of the Korean Standard Classification of Disease. We analyzed the annual number of patients according to age in 10year increments, sex, type of medical institution, and administrative division. Results: The total number of patients and number of patients undergoing ERCP per 100,000 people increased from 40,516 and 78.6 in 2013 to 47,027 and 91.3 in 2017 , respectively those aged 70 years accounted for the highest number, and the increase was the most prominent in those aged 80 years or older. Men underwent more ERCPs than women, except in younger patients ( $<40$ years). Most ERCPs were performed at tertiary and general hospitals; however, the ratios between the two types of institutions were different according to the procedure. There were different patterns of patients associated with each procedure according to the administrative division. Therapeutic ERCP was performed more frequently than diagnostic ERCP. Conclusions: Most procedures in Korea were therapeutic, and the increase in patients was most prominent in those aged 80 years or older. The utilization of ERCP increased markedly and varied depending on age, sex, type of medical institution, and region. (Gut Liver 2020;14:833-841)

Key Words: Cholangiopancreatography, endoscopic retrograde; Demography; Big data; Republic of Korea; Health services

\section{INTRODUCTION}

Endoscopic retrograde cholangiopancreatography (ERCP) is an essential endoscopic technique for various pancreatobiliary disorders. Since the introduction of ERCP in the 1960s, ${ }^{1}$ it has been recognized as the standard treatment method for choledocholithiasis, and endoscopic stent placement has replaced surgical bypass for malignant biliary obstructions. According to a recent study using inpatient database, the rate of bile duct surgery decreased dramatically, while that of therapeutic ERCP utilization increased significantly in the United States. ${ }^{2}$

The utilization of ERCP was variable, dependent on age, sex, and region. In a Canadian study, the ERCP rate increased for most women and elderly men. ${ }^{3}$ A recent analysis reported that the total rate of ERCP utilization decreased from 98.0 to 92.5 per 100,000 cases in the United States between 1998 and $2013 .{ }^{2}$ Conversely, it has significantly increased in China between 2006 and 2012 (from 4.9 to 14.4 per 100,000). ${ }^{4}$ In Korea, since its introduction in $1973,{ }^{5}$ the procedure has rapidly increased; however, to the best of our knowledge, there is limited literature on the trend of ERCP at a national scale in Korea.

This study aimed to understand the current state and to evaluate the national trend of ERCP utilization for the recent 5 years in Korea using publicly open Health Insurance Review and Assessment (HIRA) data.

\section{MATERIALS AND METHODS}

\section{Dataset}

In Korea, the National Health Insurance Service, as a single national insurer, is in charge of national health insurance for almost the entire Korean population (approximately 51 million

Correspondence to: Jaihwan Kim

Division of Gastroenterology, Department of Internal Medicine, Seoul National University Bundang Hospital, 82 Gumi-ro $173 b e o n-g i l$, Bundang-gu, Seongnam 13620, Korea

Tel: +82-31-787-7075, Fax: +82-31-787-4051, E-mail: drjaihwan@snu.ac.kr

Received on July 17, 2019. Revised on October 20, 2019. Accepted on October 29, 2019. Published online December $13,2019$.

pISSN 1976-2283 eISSN 2005-1212 https://doi.org/10.5009/gnl19249

@) This is an Open Access article distributed under the terms of the Creative Commons Attribution Non-Commercial License (http://creativecommons.org/licenses/by-nc/4.0) which permits unrestricted non-commercial use, distribution, and reproduction in any medium, provided the original work is properly cited. 
people). It is responsible for the management and enrollment of the insured and their dependents; collection of insurance contribution; and providing health insurance benefits. The HIRA reviews and assesses the healthcare costs and healthcare service quality, and it has all the data regarding the medical expenses for medical claims.

Between 2013 and 2017, the public data regarding ERCP was acquired from the HIRA database. To acquire ERCP-related data, procedural codes of the Korean Standard Classification of Disease (KCD) were used. ERCP-related KCD codes are presented in Table 1. The parameters analyzed in this study were as follows: the annual number of patients according to sex, age in 10-year increments, type of medical institutions, and administrative divisions.

To present the age-adjusted rate, the 2015 standard population data was acquired from the Korean Statistical Information Service. The Population and Housing Census of Korea conducts a survey every 5 years; 2015 was the year when the Census conducted a survey between 2013 and 2017. There were eight cities and nine provinces in the Korean Statistical Information Service dataset, and seven cities and nine provinces in the HIRA dataset; Sejong was included in the Chungnam province in the HIRA dataset. In this study, cities except Seoul, which is the capital of South Korea, were combined into the following provinces: (1) Incheon and Gyeonggi into Gyeonggi; (2) Sejong, Daejeon

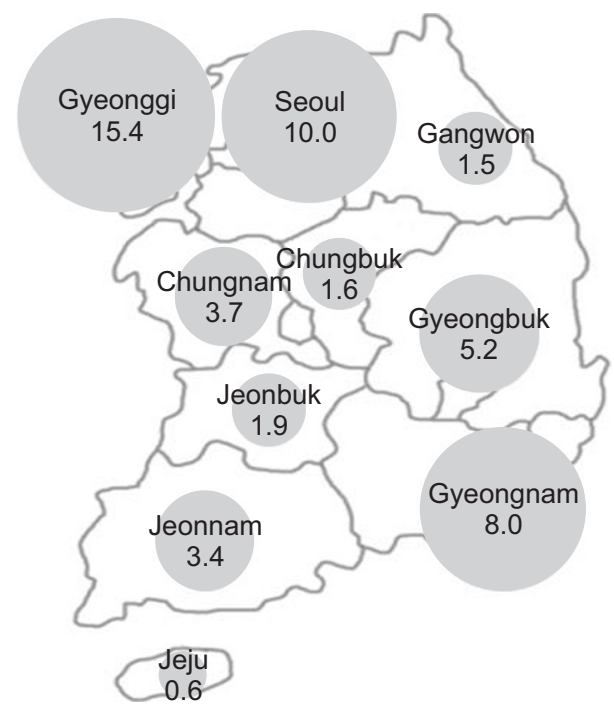

Fig. 1. The administrative divisions and populations (unit: million) in South Korea.

Table 1. ERCP-Related KCD Codes

\begin{tabular}{cllc}
\hline KCD code & \multicolumn{1}{c}{ ERCP procedures in HIRA dataset } & Categorization in this study & Purpose of procedures \\
\hline E7621 & Endoscopic retrograde cholangiopancreatography & ERCP & Diagnostic \\
E7622 & Transpapillary cholangiopancreatoscopy & & Therapeutic \\
Q7761 & Papillary sphincterotomy & EST & ERBD or ERPD \\
Q7762 & Biliary (pancreatic) drainage & Dilatation \\
Q7763 & Endoscopic biliary (pancreatic) stricture dilatation & Standard Stone Removal \\
Q7764 & Biliary (pancreatic) stone removal (basket or balloon catheter, me- & & \\
& chanical lithotripsy) & Others \\
Q7765 & Biliary (pancreatic) stone removal (electrohydraulic lithotripsy) & & \\
Q7767 & Polypectomy and tumorectomy & Foreign body removal & \\
\hline
\end{tabular}

ERCP, endoscopic retrograde cholangiopancreatography; KCD, Korean Standard Classification of Disease; HIRA, Health Insurance Review and Assessment; EST, endoscopic sphincterotomy; ERBD, endoscopic retrograde biliary drainage; ERPD, endoscopic retrograde pancreatic drainage.

Table 2. Patients Who Underwent All ERCP Procedures by Age in 10-Year Increments

\begin{tabular}{lccccc}
\hline \multirow{2}{*}{ Age, yr } & \multicolumn{5}{c}{ Year, No. (\%) } \\
\cline { 2 - 6 } & 2013 & 2014 & 2015 & 2016 & $2,792(5.9)$ \\
\hline 40 & $2,881(7.1)$ & $2,798(6.8)$ & $2,714(6.6)$ & $3,017(6.4)$ & $3,599(7.6)$ \\
$40-49$ & $3,299(8.1)$ & $3,385(8.2)$ & $3,303(8.0)$ & $7,435(7.3)$ \\
$50-59$ & $6,885(17.0)$ & $7,150(17.3)$ & $6,790(16.4)$ & $10,549(16.0)$ & $7,372(15.7)$ \\
$60-69$ & $9,347(23.1)$ & $9,448(22.8)$ & $9,371(22.7)$ & $10,949(23.3)$ & $10,809(23.0)$ \\
$70-79$ & $12,271(30.3)$ & $12,207(29.5)$ & $12,282(29.7)$ & $13,712(29.1)$ & $13,616(29.0)$ \\
$\geq 80$ & $6,008(14.8)$ & $6,518(15.8)$ & $7,054(17.1)$ & $8,437(17.9)$ & $9,251(19.7)$ \\
Total & $40,516(100.0)$ & $41,348(100.0)$ & $41,332(100.0)$ & $47,057(100.0)$ & $47,027(100.0)$ \\
\hline
\end{tabular}

ERCP, endoscopic retrograde cholangiopancreatography. 
and Chungnam into Chungnam; (3) Gwangju and Jeonnam into Jeonnam; (4) Daegu and Gyeongbuk into Gyeongbuk; and (5) Busan, Ulsan, and Gyeongnam into Gyeongnam (Fig. 1).

This study was exempt from a review by the ethical committee because it did not collect or record any personally identifiable information.

\section{Categorization of ERCP procedures and medical institu- tions}

In the HIRA database, there were nine ERCP-related procedural codes (Table 1). Of these, ERCP (E7621) was also one of the ERCP procedures in the dataset. Because the numbers of patients with E7622 (Transpapillary Cholangiopancreatoscopy), Q7765 (Biliary [Pancreatic] Stone Removal [electrohydraulic lithotripsy]), Q7766 (Polypectomy and Tumorectomy), and Q7767 (Foreign Body Removal) were too small (E7622, Q7766, Q7767-less than 1,000 patients in a year, Q7765-less than 100 patients in a year), nine codes were categorized into six in this study. Therefore, E7621 and E7622 were categorized as "ERCP," and Q7765, Q7766, and Q7767 were categorized as "Others." Consequently, six categorized procedures were ERCP, EST, ERBD or ERPD(ERBD/ERPD), Dilatation, Standard Stone Removal, and Others. Of these, ERCP was regarded as a diagnostic procedure, and other procedures were regarded as therapeutic procedures.

The types of medical institution were tertiary hospital, general hospital, hospital, and clinic. Of these, hospital and clinic were categorized into Hospital-Clinic, due to the small number of patients who received ERCP in these institutions.

\section{Statistics}

The coefficient of variation (CV) was defined as the percentage of the ratio of the standard deviation to the mean. All anal- yses and drawing plots were performed using R software v3.4.3 (R Foundation for Statistical Computing, Vienna, Austria).

Coefficient of variation $(\mathrm{CV})=\frac{\text { Standard deviation }}{\text { Mean }} \times 100$

\section{RESULTS}

\section{Number of patients based on age in 10-year increments}

The total number of patients who received ERCP increased from 40,516 in 2013 to 47,027 in 2017; during this time period,

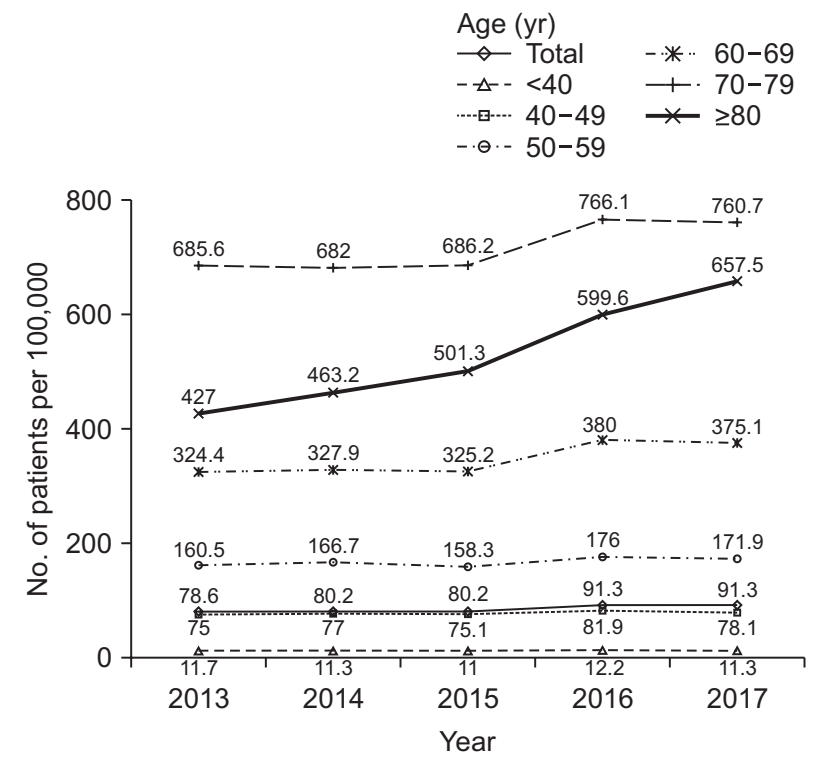

Fig. 2. Population-adjusted number of patients according to age in 10-year increments.

Table 3. Male and Female Patients per 100,000 by Age in 10-Year Increments

\begin{tabular}{|c|c|c|c|c|c|c|}
\hline \multirow{2}{*}{ Age, yr } & \multirow{2}{*}{ Sex } & \multicolumn{5}{|c|}{ Year } \\
\hline & & 2013 & 2014 & 2015 & 2016 & 2017 \\
\hline \multirow[t]{2}{*}{$<40$} & Male & 10.7 & 9.9 & 9.8 & 11.1 & 10.2 \\
\hline & Female & 12.7 & 12.8 & 12.2 & 13.4 & 12.5 \\
\hline \multirow[t]{2}{*}{$40-49$} & Male & 91.0 & 90.1 & 87.7 & 98.5 & 94.5 \\
\hline & Female & 58.5 & 63.5 & 62.1 & 64.7 & 61.1 \\
\hline \multirow[t]{2}{*}{$50-59$} & Male & 200.5 & 208.5 & 197.7 & 221.2 & 216.9 \\
\hline & Female & 119.3 & 123.6 & 117.7 & 129.4 & 125.4 \\
\hline \multirow[t]{2}{*}{$60-69$} & Male & 407.6 & 415.1 & 419.7 & 487.9 & 478.2 \\
\hline & Female & 244.5 & 244.0 & 234.4 & 276.3 & 276.0 \\
\hline \multirow[t]{2}{*}{$70-79$} & Male & 848.0 & 828.5 & 843.1 & 953.6 & 951.2 \\
\hline & Female & 553.8 & 563.1 & 558.8 & 613.9 & 606.1 \\
\hline \multirow[t]{2}{*}{$\geq 80$} & Male & 584.3 & 608.1 & 708.8 & 796.9 & 901.3 \\
\hline & Female & 356.9 & 398.7 & 408.8 & 511.7 & 548.8 \\
\hline \multirow[t]{2}{*}{ Total } & Male & 88.8 & 89.2 & 90.4 & 102.6 & 102.6 \\
\hline & Female & 69.2 & 71.9 & 70.7 & 80.8 & 80.9 \\
\hline
\end{tabular}


ERCP

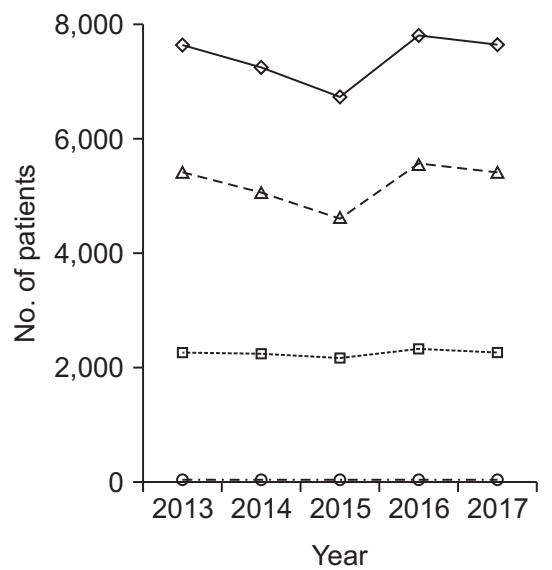

\section{Dilatation}

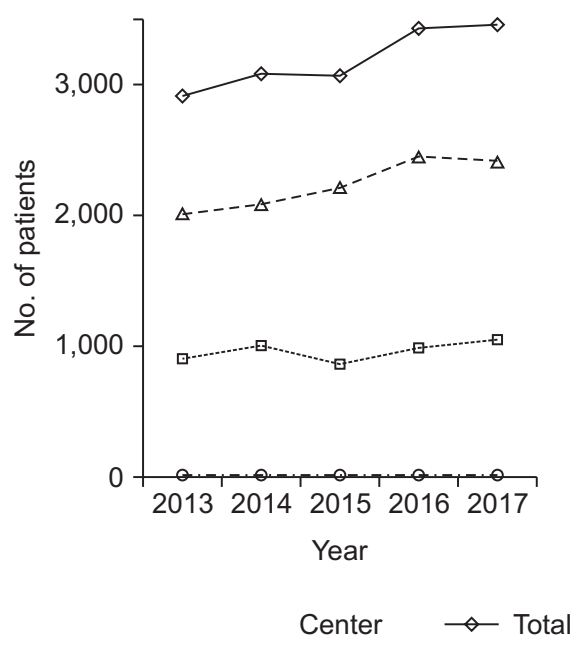

EST

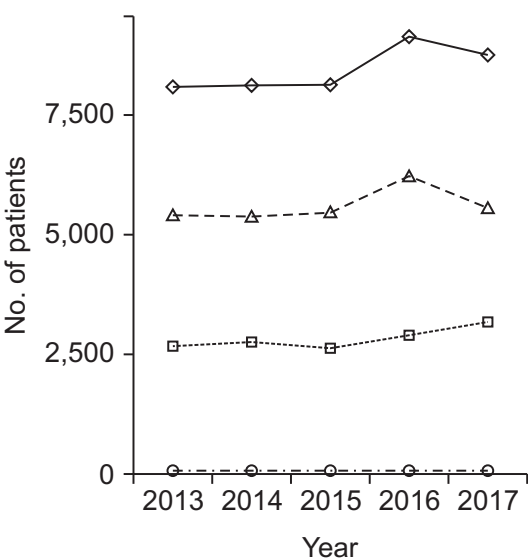

Standard Stone Removal

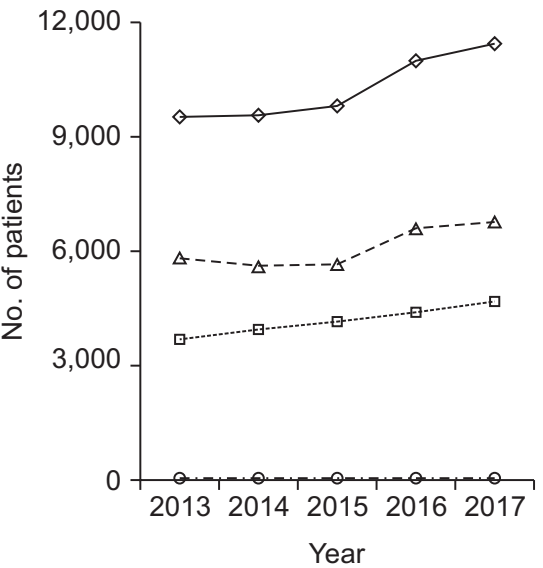

ERBD or ERPD

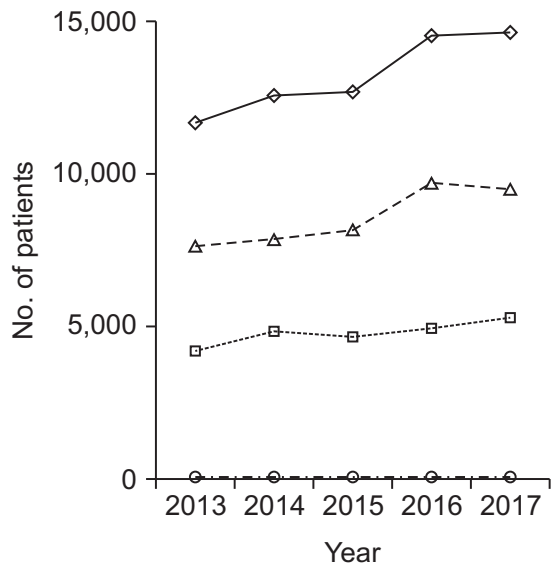

Others

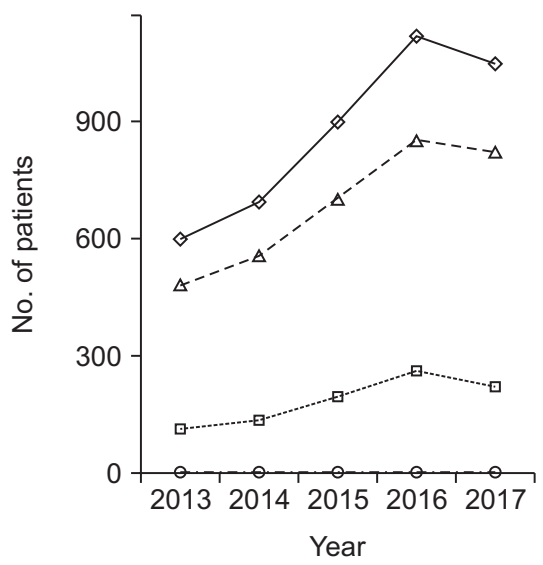

Fig. 3. Number of patients by medical institution.

ERCP, endoscopic retrograde cholangiopancreatography; EST, endoscopic sphincterotomy; ERBD, endoscopic retrograde biliary drainage; ERPD, endoscopic retrograde pancreatic drainage.

there was a notable increase between 2015 and 2016 (Table 2). It was around 41,000 between 2013 and 2015, but increased to 47,027 in 2017 . With respect to age in 10-year increments, the number of patients in the age groups 60s, 70s, and greater than 80 s, was increased. An increase in these age groups was more than 1,000 patients per group between 2015 and 2016, respectively. The 70s group had the highest number of patients, followed by the 60s group. After population adjustment, the 70 group still had the greatest number of patients per 100,000; however, the $>80$ group had the second largest number of patients per 100,000, and this group exhibited the greatest increase in patient number (Fig. 2). Males received 1.5 times more ERCPs than females, except in the group with age 40 years or less, in which more females received ERCPs than males (Table 3). Overall, there were more males than females in this study.

\section{Number of patients according to ERCP procedures and medical institutions}

Of the six procedures, ERBD/ERPD was the most common, and the Standard Stone Removal procedure was the second most common (Fig. 3). While the number of patients who underwent diagnostic procedure (ERCP) did not change significantly, the number of those who underwent therapeutic procedures (the other five procedures) increased during the study period.

According to the types of medical institutions, the ratio of tertiary hospital to general hospital was greater than two in ERCP, dilatation, and others, while it was less than two in ERBD/ERPD and Standard Stone Removal procedures. In the case of EST, the ratio was less than two in 2014 and 2017; however, it was more than two in 2013, 2015, and 2016. The number of patients who received each procedure in a hospital or clinic was less than 100 per year. 
Table 4. Patients Who Underwent All ERCP Procedures by Administrative Division

\begin{tabular}{|c|c|c|c|c|c|}
\hline \multirow{2}{*}{ Region } & \multicolumn{5}{|c|}{ Year, No. (\%) } \\
\hline & 2013 & 2014 & 2015 & 2016 & 2017 \\
\hline Seoul & $14,492(35.5)$ & $14,339(34.7)$ & $14,164(34.3)$ & 16,707 (35.5) & $16,055(34.1)$ \\
\hline Gyeonggi & $7,564(18.7)$ & $8,313(20.1)$ & 8,951 (21.7) & $10,358(22.0)$ & $10,474(22.3)$ \\
\hline Gangwon & 1,095 (2.7) & $1,190(2.9)$ & $1,181(2.9)$ & $1,272(2.7)$ & $1,299(2.8)$ \\
\hline Chungbuk & $858(2.1)$ & $889(2.2)$ & 771 (1.9) & $832(1.8)$ & 885 (1.9) \\
\hline Chungnam & $2,138(5.3)$ & $2,291(5.5)$ & $2,156(5.2)$ & $2,532(5.4)$ & 2,767 (5.9) \\
\hline Jeonbuk & $1,883(4.6)$ & $1,976(4.8)$ & $1,968(4.8)$ & $2,064(4.4)$ & 1,822 (3.9) \\
\hline Jeonnam & $2,466(6.1)$ & 2,385 (5.8) & 2,497 (6.0) & $2,734(5.8)$ & 2,765 (5.9) \\
\hline Gyeongbuk & $3,810(9.4)$ & 3,579 (8.7) & $3,576(8.7)$ & $3,870(8.2)$ & $4,004(8.5)$ \\
\hline Gyeongnam & $6,076(15.0)$ & $6,268(15.2)$ & $5,963(14.4)$ & $6,665(14.2)$ & 6,877 (14.6) \\
\hline Jeju & $432(1.1)$ & $414(1.0)$ & 415 (1.0) & $376(0.8)$ & 409 (0.9) \\
\hline Total & 40,516 (100.0) & $41,348(100.0)$ & $41,332(100.0)$ & $47,057(100.0)$ & $47,027(100.0)$ \\
\hline
\end{tabular}

ERCP, endoscopic retrograde cholangiopancreatography.

\section{Number of patients according to administrative divisions}

According to administrative divisions, Seoul had the largest number of patients, followed by Gyeonggi (Table 4). There was a continuously increasing trend in Gyeonggi during the 5-year period. After adjusting for the population, there was still the largest number of patients in Seoul per 100,000 population; the city with the second largest number of patients per 100,000 population was Jeonbuk (Fig. 4).

Although ERBD/ERPD was the most common procedure and the Standard Stone Removal procedure was the second most common in the total patient population, the pattern of procedures in each division was much different from each other (Fig. 5). The Standard Stone Removal procedure was performed relatively less frequently in Seoul; however, it was the most common procedure in Gangwon. EST, Standard Stone Removal, and ERBD/ERPD were all similarly performed in Gyeonggi. There was a rapid change in the frequency of ERBD/ERPD, Standard Stone Removal, and Dilatation in Chungbuk. EST was relatively less common in Gangwon, Chungnam, Jeonbuk, and Gyeongnam. Dilatation was relatively more common in Gyeonggi and Chungnam.

\section{Procedural count variation according to administrative divisions}

To compare each procedural count variation according to divisions, the log 10 value for the 5-year average count ratio of each division to the whole country after population adjustment was calculated. This is shown in Fig. 6. Seoul had the largest adjusted population undergoing all procedures, except the Standard Stone Removal. Jeonbuk had the largest populationadjusted number of patients undergoing the Standard Stone Removal procedure. The divisions with the smallest number of patients were different according to the procedures.

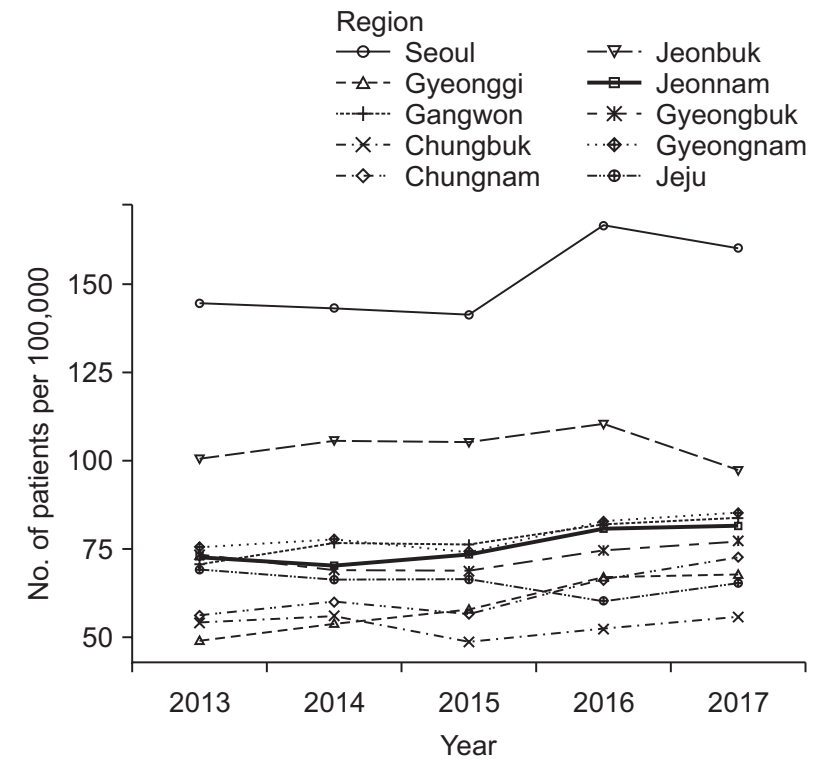

Fig. 4. Population-adjusted number of patients by administrative division.

CVs after population adjustment are presented in Table 5. Of the six procedures, others showed the largest variation $(\mathrm{CV}$, 142.9\%), and ERBD/ERPD showed the smallest variation (CV, 35.8\%) among the 10 divisions.

\section{DISCUSSION}

In Korea, the nationwide statistical data of ERCP is still limited compared with splendid achievements regarding research and technique. Recently, the number of ERCP procedures performed in Korea between 2010 and 2014 was reported by the Korean Pancreatobiliary Association, and a comparison of the annual rate of ERCP performed among the counties was 
Total (2013-2017)

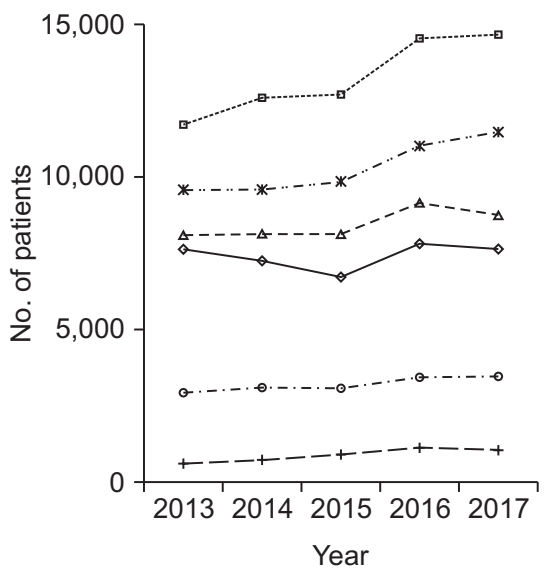

Gangwon (2013-2017)

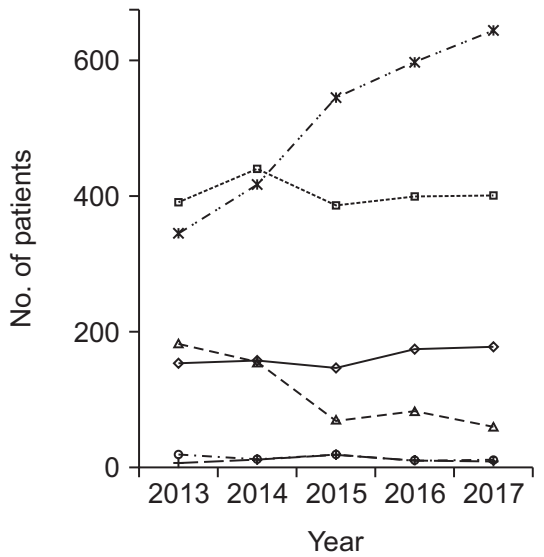

Jeongbuk (2013-2017)

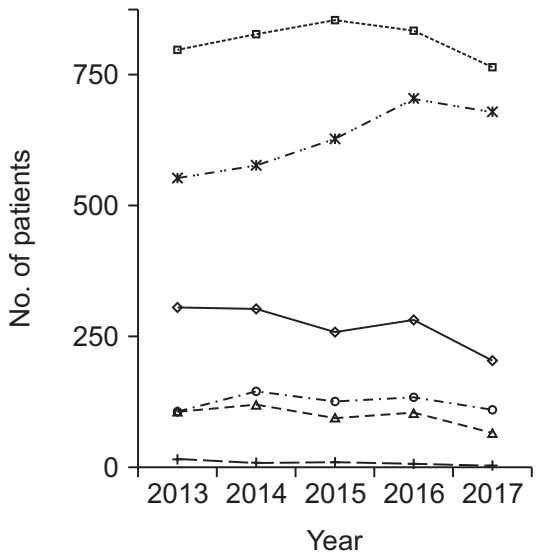

Seoul (2013-2017)

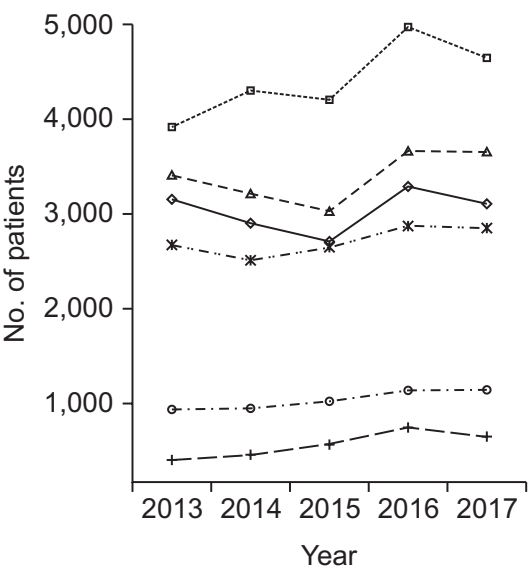

Chungbuk (2013-2017)

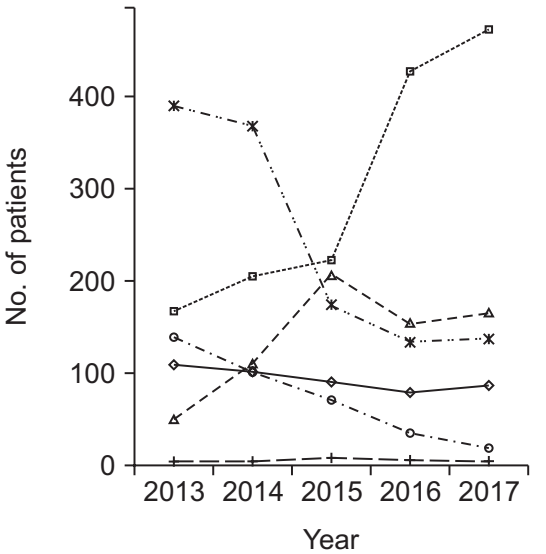

Jeonnam (2013-2017)

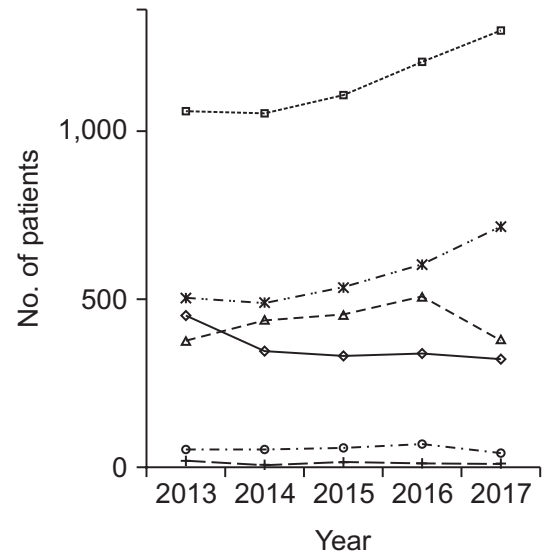

Gyeonggi (2013-2017)

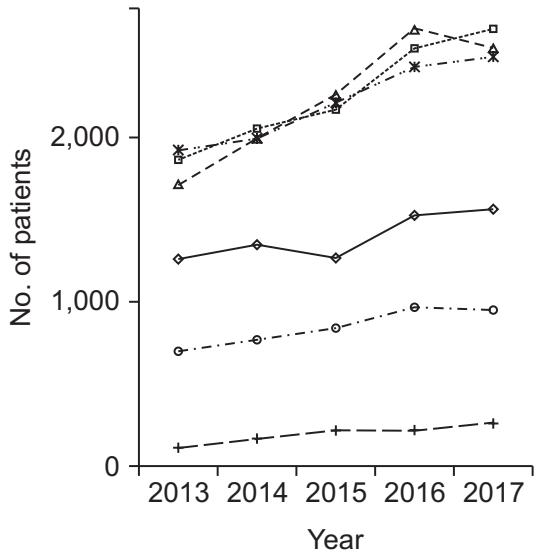

Chungnam (2013-2017)

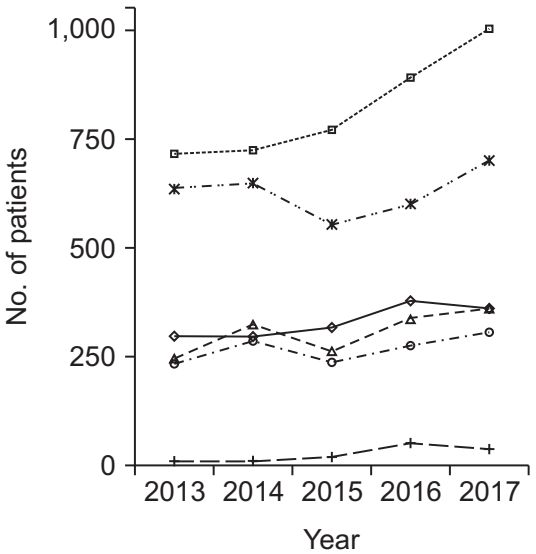

Gyeongbuk (2013-2017)

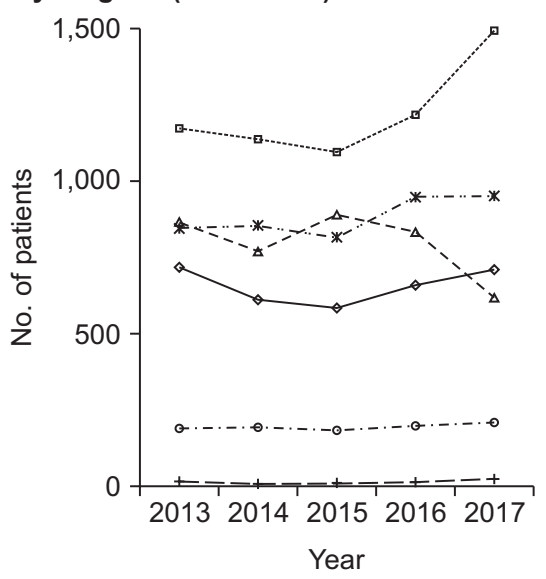

Procedures

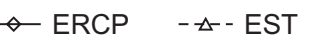

.. ERBD or ERPD

-.๑-- Dilatation

Standard Stone Remova

Others

Fig. 5. Trends of endoscopic retrograde cholangiopancreatography procedures in each administrative division.

ERCP, endoscopic retrograde cholangiopancreatography; EST, endoscopic sphincterotomy; ERBD, endoscopic retrograde biliary drainage; ERPD, endoscopic retrograde pancreatic drainage.

presented. ${ }^{6}$ According to the study, the number of ERCP has increased from 40,162 to 47,983, and the annual ERCP rate was 82.6 per 100,000 in 2010 . When compared with other countries, the annual ERCP rate in Korea was less than those in Austria, ${ }^{7}$ Canada, ${ }^{3}$ and the Netherlands. ${ }^{8}$ On the other hand, it was greater than those in England, ${ }^{9}$ Sweden, ${ }^{10}$ and China. ${ }^{4}$ Although the study reported the first nationwide rate of ERCPs performed in Korea, the main content was the survey results for endoscopists registered in the Korean Pancreatobiliary Association. Therefore, it lacked information regarding the nationwide trend of ERCP in 


\section{Gyeongnam (2013-2017)}

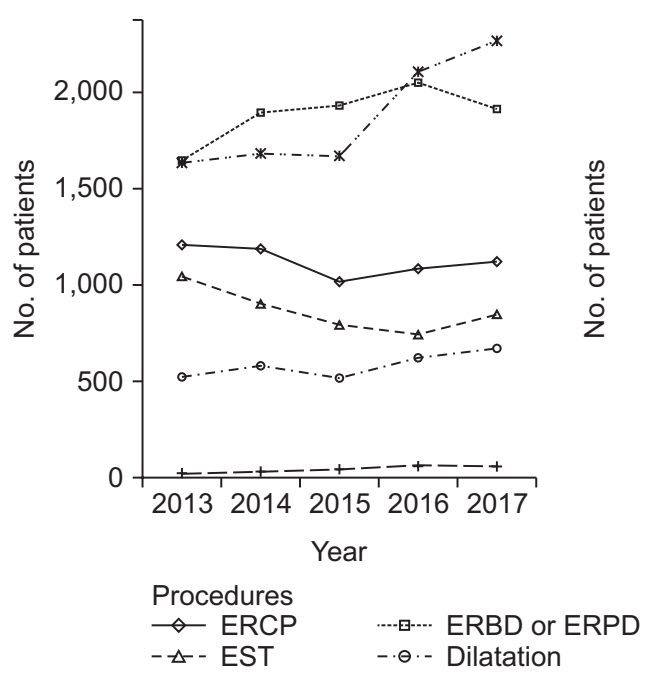

Jeju (2013-2017)

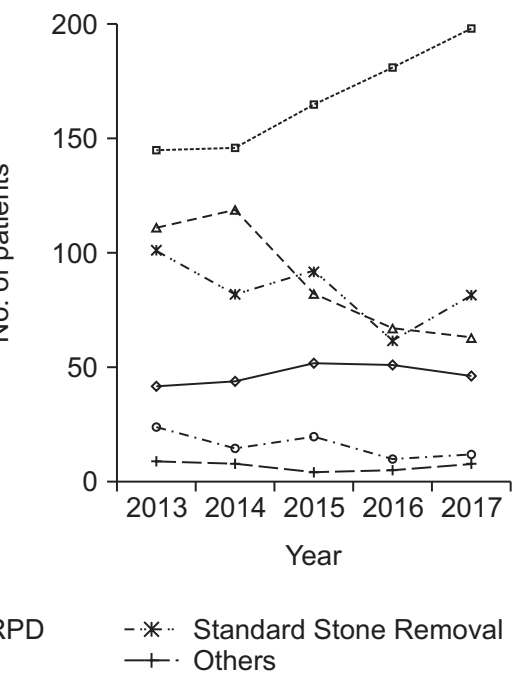

Fig. 5. Continued.

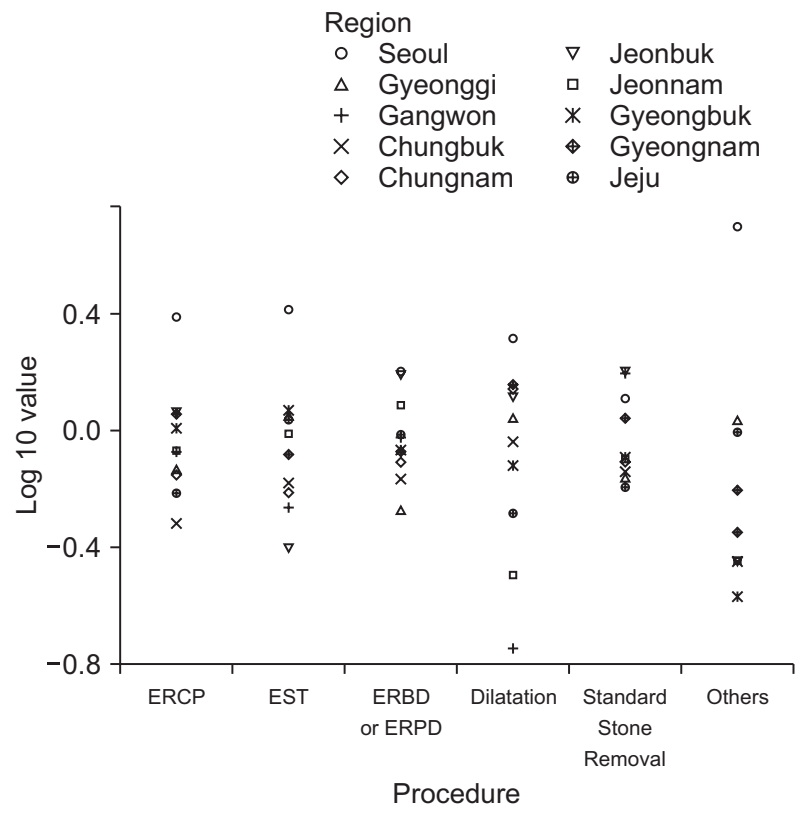

Fig. 6. Five-year average procedural count ratio of each division to the whole country after population adjustment.

ERCP, endoscopic retrograde cholangiopancreatography; EST, endoscopic sphincterotomy; ERBD, endoscopic retrograde biliary drainage; ERPD, endoscopic retrograde pancreatic drainage.

detail. In this aspect, the current study tried to analyze the trend of ERCPs performed in the recent 5 years, based on the publicly available dataset. Because the current study reported the number of patients instead of the number of procedures, there was a slight difference in the number of ERCP and annual rates compared with the previous study.

According to our study, the number of patients with ERCP per 100,000 has increased from 78.6 in 2013 to 91.3 in 2017.
It was comparable to that of the United States in 2013, which was $92.5,{ }^{2}$ but much more than that of China in 2014 (14.4 procedures/100,000). ${ }^{4}$ The number of patients markedly increased between 2015 and 2016, and it was prominent in the older age groups. The reasons for this marked increase were not clear, although the change of cost, the change of number of endoscopists or medical institutions, or the aging society were all suspected to be reasons for the increase.

ERCP was commonly performed in elderly patients and least performed in patients under the age of 40 years. This indicates that elderly people may need more procedures than younger people. The increasing age of the population undergoing ERCP is in line with previous studies; ${ }^{3,11}$ this is likely attributable to the fact that gallstones and pancreatobiliary malignancies are more common in elderly people. Moreover, the dramatic increase in patients older than 80 years may suggest a rapidly aging society in Korea. ${ }^{12}$ Preference for less invasive treatment in older patients and steady increase of malignancy could also contribute to this trend, which was already suggested in a previous study. ${ }^{3}$ The development of sedation and periprocedural care may also contribute to this trend.

Regarding sex difference, there were more male patients, and female sex was dominant only in patients less than 40 years. This pattern is much different in Western countries. In a study from Canada, ERCPs were performed 1.7 times more in women than in men; and female predominance had been steady throughout the 25-year period of analysis. ${ }^{3}$ Similarly, ERCPs were also 1.7 times more common in females than in males in the United States for the past 15-year period. Men were dominant in age less than 40 years, while women were dominant in age over 40 years. ${ }^{11}$ Several factors may be attributable to this difference, e.g., the relatively low proportion of cholesterol stone 
Table 5. Five-Year Average Status of Each ERCP Procedure after Population Adjustment (per 100,000 Population) by Administrative Division

\begin{tabular}{lcccccc}
\hline & $\begin{array}{c}\text { ERCP } \\
(\text { E7621, E762) }\end{array}$ & $\begin{array}{c}\text { EST } \\
(\text { Q7761) }\end{array}$ & $\begin{array}{c}\text { ERBD or ERPD } \\
(\text { Q7762) }\end{array}$ & $\begin{array}{c}\text { Dilatation } \\
\text { (Q7763) }\end{array}$ & $\begin{array}{c}\text { Standard Stone } \\
\text { Removal (Q7764) }\end{array}$ & $\begin{array}{c}\text { Others } \\
\text { (Q7765, Q7766, Q7767) }\end{array}$ \\
\hline Mean & 12.34 & 13.03 & 27.62 & 5.02 & 20.99 & 1.12 \\
SD & 6.9 & 8.1 & 9.9 & 2.9 & 7.7 & 1.6 \\
CV (\%) & 55.9 & 62.2 & 35.8 & 57.8 & 36.7 & 142.9 \\
Max & 30.3 & 33.9 & 44.0 & 10.4 & 33.6 & 5.6 \\
Min & 5.9 & 5.2 & 14.6 & 0.9 & 13.4 & 0.3 \\
Max-Min & 24.4 & 28.7 & 29.4 & 9.5 & 20.2 & 5.3 \\
Max/Min & 5.1 & 6.5 & 3.0 & 11.6 & 2.5 & 18.7 \\
\hline
\end{tabular}

ERCP, endoscopic retrograde cholangiopancreatography; EST, endoscopic sphincterotomy; ERBD, endoscopic retrograde biliary drainage; ERPD, endoscopic retrograde pancreatic drainage; CV, coefficient of variation; Max, maximum; Min, minimum.

in Korea compared with Western countries. ${ }^{13}$ On the other hand, oriental cholangiohepatitis with pigmented stone does not show female predominance (equal sex incidence) ${ }^{14}$ In addition, biliary tract cancer is more common amongst males in Korea, while it is generally an uncommon malignancy in Western countries. ${ }^{15}$

Most procedures were performed in tertiary and general hospitals; however, there were different ratios between these two medical institutions. This suggests that these procedures may relatively be difficult to perform; ${ }^{16}$ in general, difficult or rare procedures are more likely performed in tertiary hospitals. The difference in the number of procedures performed between hospital and clinic was negligible.

With respect to the ratio of therapeutic to diagnostic ERCP, the average annual number of therapeutic ERCPs performed was far higher than that of diagnostic ERCPs, which was also seen in other studies. ${ }^{2-4,17,18}$ Moreover, therapeutic procedures continuously increased throughout the 5-year period; however, there was no increase in the number of diagnostic ERCPs performed. Nonetheless, the frequency of diagnostic ERCP may still seem very high, with about 7,000 diagnostic ERCPs performed per year. It is difficult to comprehend all information about each procedure with the publicly available HIRA data. However, Korean endoscopists may choose these codes in the event of selective biliary cannulation failure. A previous study showed that in the United States, the frequency of therapeutic ERCP was 5.9 times higher than that of diagnostic ERCP in 2013, which is similar to the findings of this study. ${ }^{17}$

Among the 10 administrative divisions, Seoul and Jeonbuk had a much greater adjusted numbers of patients per 100,000 than other divisions. In case of Seoul, it can be guessed that many patients from other divisions were treated in Seoul, and there were many tertiary or general hospitals. There were large differences of proportions of procedures according to the administrative divisions. It was not clear that the differences came from the different diseases or different patterns of preferred procedures or medical reimbursement.

In this study, CV of others was much bigger than the Standard Stone Removal or ERBD/ERPD. CV is known to be good for comparing the variability of each variable with different arithmetic means. A more accurate comparison could be made by comparing the CVs than by comparing the standard deviation, ${ }^{19}$ because a higher variability is usually expected when the mean increases, and the CV is a measure that accounts for this variability. Consequently, a higher CV may indicate a higher variability according to the divisions, and the outcome of this study showed that the variability of others was higher than those of Stone Removal or ERBD/ERPD. It indicates that electrohydraulic lithotripsy, endoscopic papillectomy, or foreign body removal is variably performed according to the administrative divisions; however, ERBD/ERPD or Standard Stone Removal is a more generalized procedure, regardless of the divisions. ${ }^{16}$

This study has several limitations. First, the number of procedures performed may be underestimated because this present study reported the number of patients instead of the number of procedures, and only the main procedure code was recorded in the HIRA dataset. Second, the dataset was based on the address of the medical institution, instead of the address of the patient. Therefore, our study may not fully represent the number of patients requiring ERCP in each division. Third, the dataset was based on the publicly available HIRA open data; therefore, we could not identify detailed information about the procedures, such as diagnosis, success, and complication rates. Fourth, only one code can be entered regardless of the number of procedures performed. For example, if a stent is inserted after the removal of stones, only one code can be selected: Standard Stone Removal versus ERBD or ERPD. Therefore, there may be inherent bias toward the endoscopist's preference at the time of code entry. Due to the inherent limitations of big data research, the study cannot be completely free from such bias. Finally, Sejong city was incorporated into Chungcheongnam-do (South Chungcheong province) when we calculated the population because Sejong city was included in Chungnam province in HIRA dataset, although it is a different administrative division from Chungcheongnam-do. However, the proportion of population of Sejong city in Korea was $0.41 \%$ in 2015 and it is not supposed to significantly affect the outcomes of this study. 
In conclusion, our analysis shows that ERCP has been increasingly performed in Korea. It was mainly performed in elderly patients, with a marked increase in those older than 80 years. Men underwent ERCPs more than women, except in the young population (<40 years). Most ERCPs were performed in tertiary and general hospitals, although the ratios between the two types of institutions were different according to the procedure. Most procedures were therapeutic, and the procedures were largely variable depending on the administrative divisions.

\section{CONFLICTS OF INTEREST}

No potential conflict of interest relevant to this article was reported.

\section{AUTHOR CONTRIBUTIONS}

Study concept and design: J.M.P., J.K. Data acquisition \& data analysis and interpretation: J.M.P., J.K. Drafting of the manuscript: J.M.P., J.K. Critical revision of the manuscript for important intellectual content: C.D.K., J.C.L., J.H.H. Statistical analysis: J.M.P., J.K. Study supervision: J.K.

\section{ORCID}

Jin Myung Park

Chang Don Kang

Jong-chan Lee

Jin-Hyeok Hwang

Jaihwan Kim https://orcid.org/0000-0002-8798-0587

https://orcid.org/0000-0002-5779-1144

https://orcid.org/0000-0001-6590-2353

https://orcid.org/0000-0002-5643-8461

https://orcid.org/0000-0003-0693-1415

\section{REFERENCES}

1. McCune WS, Shorb PE, Moscovitz H. Endoscopic cannulation of the ampulla of Vater: a preliminary report. Ann Surg 1968;167:752-756.

2. Huang RJ, Thosani NC, Barakat MT, et al. Evolution in the utilization of biliary interventions in the United States: results of a nationwide longitudinal study from 1998 to 2013. Gastrointest Endosc 2017;86:319-326.

3. Moffatt DC, Yu BN, Yie W, Bernstein CN. Trends in utilization of diagnostic and therapeutic ERCP and cholecystectomy over the past 25 years: a population-based study. Gastrointest Endosc. 2014;79:615-622.

4. Hu LH, Xin L, Liao Z, et al. ERCP development in the largest developing country: a national survey from China in 2013. Gastrointest Endosc 2016;84:659-666.

5. Kim HG. Korean Pancreatobiliary Association 20th Anniversary.
Seoul: Korean Pancreatobiliary Association, 2015.

6. Ahn DW, Han JH, Kim HJ, et al. Practice of endoscopic retrograde cholangiopancreatography in Korea: results from a national survey. Korean J Pancreas Biliary Tract 2019;24:21-30.

7. Kapral C, Duller C, Wewalka F, Kerstan E, Vogel W, Schreiber F. Case volume and outcome of endoscopic retrograde cholangiopancreatography: results of a nationwide Austrian benchmarking project. Endoscopy 2008;40:625-630.

8. Ekkelenkamp VE, de Man RA, Ter Borg F, et al. Prospective evaluation of ERCP performance: results of a nationwide quality registry. Endoscopy 2015;47:503-507.

9. Bodger K, Bowering K, Sarkar S, Thompson E, Pearson MG. Allcause mortality after first ERCP in England: clinically guided analysis of hospital episode statistics with linkage to registry of death. Gastrointest Endosc 2011;74:825-833.

10. Enochsson L, Swahn F, Arnelo U, Nilsson M, Löhr M, Persson G. Nationwide, population-based data from 11,074 ERCP procedures from the Swedish Registry for Gallstone Surgery and ERCP. Gastrointest Endosc 2010;72:1175-1184.

11. Mazen Jamal M, Yoon EJ, Saadi A, Sy TY, Hashemzadeh M. Trends in the utilization of endoscopic retrograde cholangiopancreatography (ERCP) in the United States. Am J Gastroenterol 2007;102:966-975.

12. Development of EC-oa. OECD statistical information system [Internet]. Paris: OECD [cited 2019 Dec 29]. Available from: https:// stats.oecd.org/.

13. Feldman M, Friedman L, Brandt L. Sleisenger and Fordtran's gastrointestinal and liver disease. 9th ed. Philadelphia: Saunders, 2010.

14. Tsui WM, Lam PW, Lee WK, Chan YK. Primary hepatolithiasis, recurrent pyogenic cholangitis, and oriental cholangiohepatitis: a tale of 3 countries. Adv Anat Pathol 2011;18:318-328.

15. Kim BW, Oh CM, Choi HY, Park JW, Cho H, Ki M. Incidence and overall survival of biliary tract cancers in South Korea from 2006 to 2015: using the National Health Information Database. Gut Liver 2019;13:104-113.

16. Ang TL, Cheng J, Khor JL, et al. Guideline on training and credentialing in endoscopic retrograde cholangiopancreatography. Singapore Med J 2011;52:654-657.

17. Ahmed M, Kanotra R, Savani GT, et al. Utilization trends in inpatient endoscopic retrograde cholangiopancreatography (ERCP): a cross-sectional US experience. Endosc Int Open 2017;5:E261E271.

18. Glomsaker T, Søreide K, Aabakken L, Søreide JA. A national audit of temporal trends in endoscopic retrograde cholangiopancreatography in Norway. Scand J Gastroenterol 2011;46:116-121.

19. Rosner B. Fundamentals of biostatistics. 7th ed. Boston: Brooks/ Cole, Cengage Learning, 2011. 